\title{
FOLIC ACID TREATMENT OF ANTICONVULSANT-INDUCED HYPERHOMOCYSTEINEMIA
}

The prevalence of hyperhomocysteinemia (HHcy) in 123 childhood epilepsy patients treated with antiepileptic drugs (AED) and the effect of folic acid supplements (1 mg/day) on plasma Hcy levels were determined in a study at three regional hospitals and pediatric centers in Austria. HHcy $(>10.4 \mathrm{mcmol} / \mathrm{L})$ was present in $19(15.5 \%)$ patients. Those with HHcy were older $(13.7+/-4$ vs $11.0+/-3.9$ years $)$ and had lower folate and cobalamin concentrations. HHcy and low folate correlated with polytherapy and long duration AED therapy. A significant increase of folate and decrease of Hcy levels were observed in 10 patients with HHcy who received folic acid supplements, in a 3-month double-blind randomized, placebo-controlled trial, whereas levels were unchanged in 9 with HHcy who received placebo. Total Hcy levels were not affected by polymorphisms in the methylene tetrahydrofolate reductase gene. (Huemer $M$, Ausserer $B$, Graninger $G$ et al. Hyperhomocysteinemia in children treated with antiepileptic drugs is normalized by folic acid supplementation. Epilepsia October 2005;46:1677-1683). (Reprints: Dr M Huemer, Landeskrankenhaus Feldkirch, Departent of Pediatrics, Carinagasse 47, 6800 Feldkirch, Austria).

COMMENT. Children taking antiepileptic drugs (AEDs) for prolonged periods should be monitored for folate and homocysteine plasma levels. Hyperhomocysteinemia and folate depletion are more prevalent in patients receiving polytherapy. The long-term effects of folic acid supplements in patients taking AEDs requires further study, particularly in relation to the optimal dose and effects on seizure control, cardiovascular disease, and cognitive function.

The significance of folic acid for epilepsy patients is reviewed by Moore JL in Epilepsy \& Behavior Sept 2005;7:172-181. In the 1960s, folic acid was considered epileptogenic by some neurologists, and supplements were used with caution in epilepsy patients. The benefits and risks of folic acid to the nervous system were reviewed by Reynolds EH in $\mathbf{J}$ Neurol Neurosurg Psychiatry 2002;72:567-571. Now, the beneficial effects of folic acid in the prevention of certain neurologic disorders, especially neural tube defects, are well known, and supplementation is common practice. Case reports of seizure exacerbations by folic acid have led to the emergence of anticonvulsants with folic acid antagonistic effects, such as lamotrigine.

\section{OUTCOME OF CRYPTOGENIC EPILEPSY}

Medical records of 60 children ( $53 \%$ males) with cryptogenic epilepsy, seen over a 12 -year period and treated at Montreal Children's Hospital, McGill University, Canada, were reviewed retrospectively to identify clinical and predictive features of outcome. Mean age of initial seizure was 7.7 years (range 2.3-16.4 years). Febrile seizures had preceded the onset of epilepsy in $11(18.3 \%)$, of which 7 were simple in type and 4 complex. Seizure types at presentation were generalized in $22(35.7 \%)$, only 1 a typical absence; partial with secondary generalization in $20(33.3 \%)$, complex partial in $17(28.3 \%)$, and simple partial (1). Physical examination was normal in 46 (76.7\%), 9 (15\%) were developmentally delayed at onset, 38 